\title{
30 Jahre dermatologische Radiotherapie
}

\section{Years of Radiation Treamtent in Dermatology}

\section{Zusammenfassung}

Die dermatologische Radiotherapie hat in den letzten 30 Jahren an Bedeutung verloren, ist aber nicht ganz verschwunden und sollte immer noch, zumindest in einzelnen Zentren, aufrecht erhalten werden. Denn bei zunehmender Überalterung der Bevölkerung ist diese Therapieform bei älteren Leuten immer noch die beste Alternative zur chirurgischen Therapie und eignet sich insbesondere für Tumore im Gesichtsbereich in ausgezeichneter Weise.

\section{Abstract}

Dermatologic Radiotherapy has reached a certain age and lost some of its importance. It is nevertheless worthwhile to maintain and develop a modern and safe dermatologic radiotherapy in at least some centers of excellence. It is, apart from dermatosurgery, an excellent und helpful therapeutic instrument in widespread malignant skin leasions, mainly in the face and especially in elderly patients.
Bis in die sechziger Jahre war die dermatologische Radiotherapie weit verbreitet, sowohl in Universitätskliniken als auch bei den niedergelassenen Dermatologen. Erste Anzeichen eines Rückgangs gab es schon in dieser Zeit, denn die chirurgischen Verfahren haben sich in der Dermatologie mehr und mehr entwickelt. Bereits vorgängig wurden Medikamente wie Kortikosteroide und Antibiotika in die Dermatotherapie eingeführt, mit welchen die verschiedensten entzündlichen Dermatosen behandelt werden konnten. Es ist auch zu beachten, dass gerade diese gutartigen Dermatosen einen großen Anteil am Krankengut der dermatologischen Radiotherapie gehabt haben. Oft war ihre Anzahl größer als die der bestrahlten Hauttumore. Weiter kommt hinzu, dass in den sechziger Jahren auch eine ausgesprochene Angst vor Strahlenenergie, z.B. im Zusammenhang mit Atomenergie, bestand. Die Ausbildung in Strahlentherapie wurde mehr und mehr vernachlässigt. Röntgengeräte für die Grenz- und Weichstrahlentherapie wurden immer weniger hergestellt und die Kontrollkosten für diejenigen, die ein Röntgengerät betrieben, stiegen an, in gewissen Ländern kam es auch zur Erhöhung der
Versicherungsgebühren. Schließlich ist auch daran zu denken, dass insbesondere bei gutartigen Hautveränderungen mehrmalige Bestrahlungen im Interesse des Patienten durchgeführt wurden und es daraufhin zu chronischen Röntgennebenwirkungen mit entsprechenden Folgen gekommen ist. Aus all den genannten Ausführungen wird verständlich, weshalb die Indikationen für eine dermatologische Radiotherapie, insbesondere gutartiger Veränderungen, deutlich zurückgegangen ist.

Auf der anderen Seite muss betont werden, dass die Radiotherapie, neben den chirurgischen Verfahren, immer noch eine der best untersuchten Therapiemethoden darstellt. Zu erinnern ist, dass der erste Patient, den W. C. Röntgen im Jahre 1900 bestrahlte, an einem Spinaliom der Nase litt. Seither haben sich unsere Erkenntnisse bezüglich Strahlenphysik und Strahlenbiologie erweitert. Zu erinnern ist an die Entwicklung der Fraktionierung der Röntgenstrahlen, der „Nominal Standard Dose“ (NSD) aber auch die Entwicklung der „Time Dose Fractionation“(TDF)-Faktoren. Auch die Wirkung kleinster Strahlendosen und die Theorie 
der deterministischen und non-deterministischen Strahleneffekte und unsere Kenntnisse bezüglich der Mechanismen akuter und chronischer Strahlenwirkungen sind besser bekannt.

Die Literatur der dermatologischen Radiotherapie wurde bis in die sechziger Jahre praktisch von Dermatologen bestritten. Es ist das Verdienst von Braun-Falco und Lukacs (Abb.1) sowie Goldschmidt (Abb. 2), in dieser wichtigen Zeit Monographien zu diesem Thema herausgegeben zu haben. $Z u$ erwähnen ist auch, dass neben diesen Monographien in verschiedenen Ländern die dermatologische Radiotherapie weiter betrieben und gelehrt wurde, trotz der oben erwähnten Gründe des Rückgangs. Später kamen weitere Bücher zu diesem Thema heraus, so von Goldschmidt und Panizzon (Abb.3), Peter und Plewig (Abb.4) und schließlich von Panizzon und Cooper (Abb.5). Insbesondere zu erwähnen ist, dass anlässlich der Kongresse der „American Academy of Dermatology“ (AAD) die dermatologische Radiotherapie bis heute immer noch auf dem Programm steht und Kurse angeboten werden. Die „European Academy of Dermatology and Venerology“ (EADV) ermöglicht Vorträge über die Röntgentherapie leider nur noch im Rahmen des Symposiums „Non Melanoma Skin Cancer“. Ähnlich ist die Situation bei den „Journées dermatologiques de Paris“ (JDP), wo nach einem Stillstand die Radiotherapie bei den konservativen Therapiemaßnahmen wieder Eingang in das Programm gefunden hat. Ebenso beim Kongress der „Deutschen Dermatologischen Gesellschaft“ (DDG). Hier ist die Radiotherapie ebenfalls nur im Rahmen der „physikalischen Behandlungsmethoden" vorgesehen. Bei uns in der Schweiz ist die Ausbildung in dermatologischer Radiotherapie im Kurs „Physikalische Therapiemaßnahmen in der Dermatologie“ alle zwei Jahre obligatorisch zur Erlangung des Facharzttitels Dermatologie und Venerologie. Dieser beinhaltet zwei Tage Ausbildung in Strahlenbiologie/Strahlenschutz/Strahlentherapie. Der Rückgang der dermatologischen Radiotherapie führte so weit, dass nur noch wenige Universitätszentren die dermatologische Radiotherapie selbst durchführen: in der Schweiz in Zürich, Basel und Bern, in Deutschland in der Fachklinik Hornheide/Münster und in den Universitätskliniken in München und in Ulm, in Frankreich lediglich in der Universitätsklinik in Bordeaux, in den USA in keiner Universitätshautklinik mehr, einzig noch in einzelnen dermatologischen Praxiszentren, in Italien in der Universitätsklinik in Mailand, in England wird die Radiotherapie nicht mehr von Dermatologen durchgeführt, ebenso in Österreich. Erhalten geblieben sind noch gewisse Universitätszentren in den osteuropäischen Ländern, in Südamerika, in Asien und in Australien (Brisbane, Geelong, Melbourne, Perth, Sydney).

Es scheint uns wichtig, dass in jedem Land mindestens ein Zentrum mit Kompetenz in diesem Fachbereich bestehen bleibt, denn wie im Nachfolgenden auszuführen ist, gibt es auch heute noch wichtige Indikationen für eine dermatologische Radiotherapie, auch wenn diese Liste gegenüber früher kleiner geworden ist. Insbesondere möchten wir auf die Bedeutung der dermatologischen Radiotherapie bei Tumorerkrankungen und hier vor allem bei älteren Leuten hinweisen. Die Radiotherapie ist und bleibt hier die beste Alternative zur Chirurgie. Innerhalb der Dermatosen hat sich die dermatologische Radiotherapie erhalten können, vor allem die nebenwirkungsarmen Grenzstrahlen, bei therapieresistenten Fällen oder besonderen Lokalisationen, z. B. der Kopfboden oder Fingernägel bei Psoriasis vulgaris im Rah-

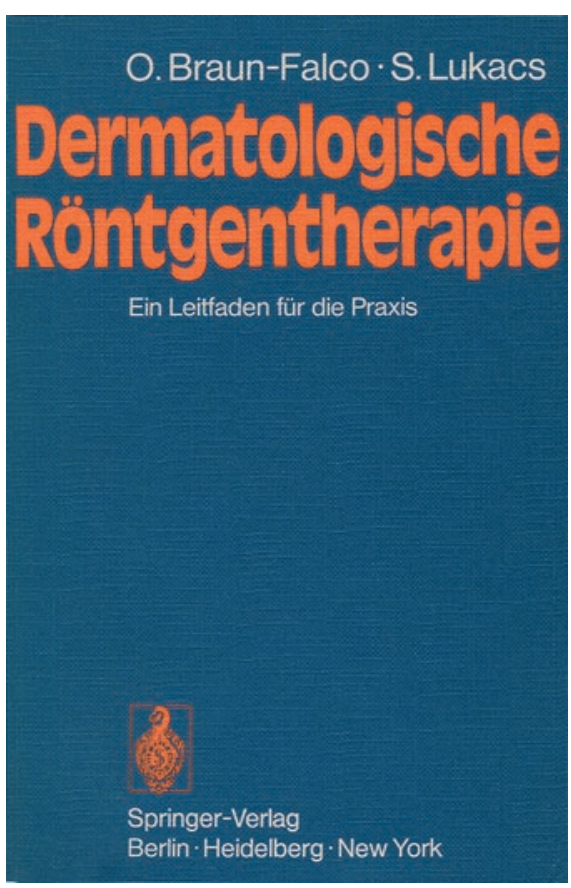

Abb. 1 O. BraunFalco, S. Lukacs: Dermatologische Röntgentherapie. Heidelberg: Springer, 1973.

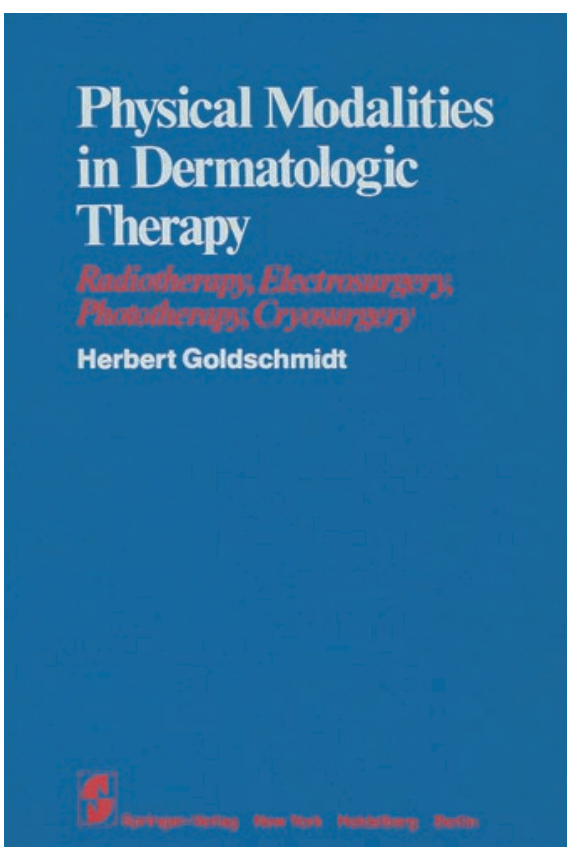

Abb. 2 H. Goldschmidt: Physical Modalities in Dermatologic Therapy. Heidelberg: Springer, 1978.

men der chronischen verselbstständigten Ekzeme, und allenfalls bei selteneren entzündlichen Veränderungen wie schmerzhaften Ulcera cruris oder ein Pemphigus chronicus familiaris HaileyHailey. Hyperplastische Hautveränderungen wie Hämangiome werden kaum mehr bestrahlt, ebensowenig die Induratio penis plastica oder die vulgären Warzen. Hingegen sind benigne lymphozytäre Hyperplasien, d.h. das Lymphozytom, immer noch eine mögliche Indikation.

Innerhalb der malignen Hauttumoren können kurativ behandelt werden die ausgedehnten aktinischen Keratosen, die ebenfalls sehr gut auf Grenzstrahlen ansprechen und auch heute noch eine längere Rezidivfreiheit aufweisen als z.B. 5-Fluorouracil (5-FU), Imiquimod oder Diclofenac. Auch der Morbus Bowen größeren Ausmaßes stellt eine sehr gute Indikation dar. Basalio- 


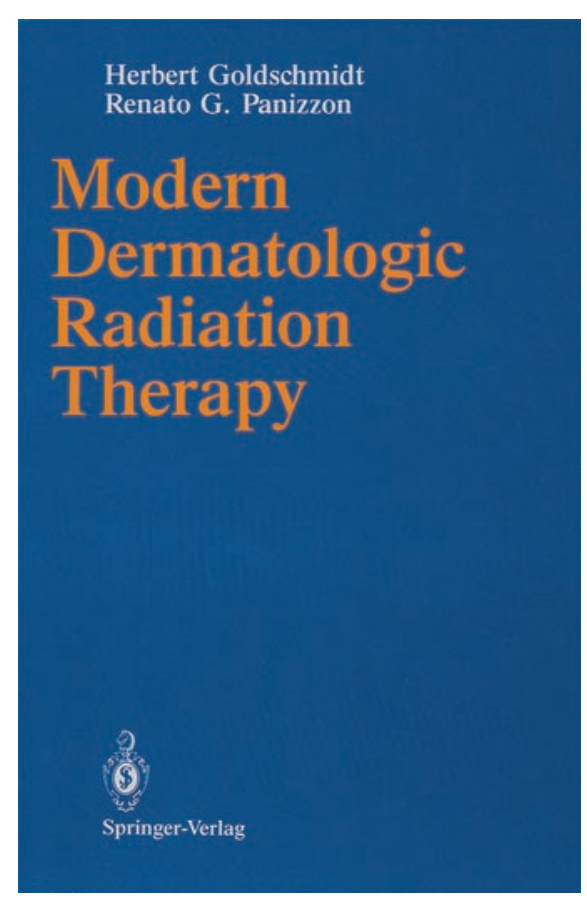

Abb. 3 H. Goldschmidt, R. G. Panizzon: Modern Dermatologic Radiation Therapy. Heidelberg: Springer, 1991.

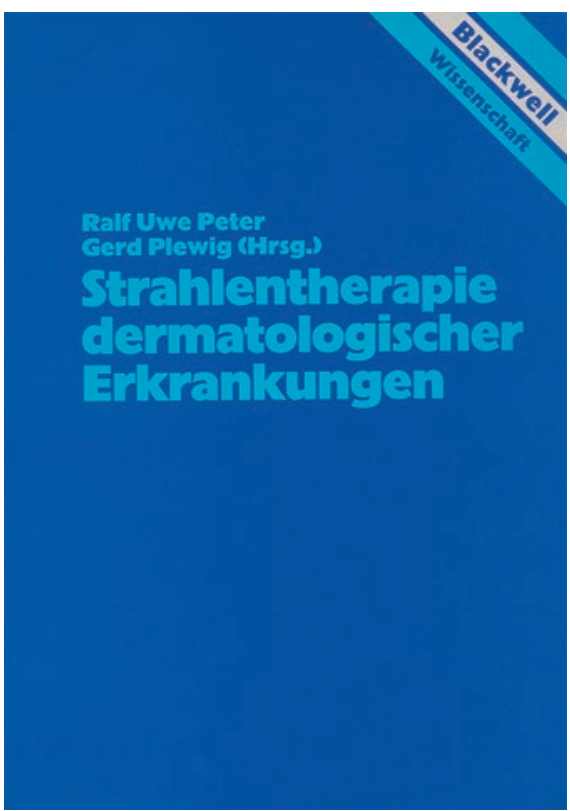

Abb. 4 R. U. Peter, G. Plewig: Strahlentherapie dermatologischer Erkrankungen. Berlin: Blackwell, 1996.

me und Spinaliome mittlerer Größe (1,5 bis 4 Zentimeter) und im Gesichtsbereich stellen nach wie vor die häufigste und wichtigste Indikation, insbesondere bei älteren Leuten, dar. Ebenfalls kurativ behandelt werden können die Lentigo maligna und das Lentigo maligna Melanom. In diese Kategorie gehört auch das Merkelzellkarzinom, das strahlensensibel ist, gelegentlich aber ein kombiniertes Verfahren zusammen mit der Chirurgie bedingt. Zu erwähnen sind auch isolierte B-Zell-Lymphome, die sehr gut strahlensensibel sind. Als zweite Gruppe möchten wir die Hauttumoren, die palliativ behandelt werden können, erwähnen. So vor allem die übrigen B-Zell-Lymphome, aber auch die T-Zell-Lymphome, das Kaposi-Sarkom, Melanom-Metastasen, leukämische Hautinfiltrate und Hautadnextumoren. Nicht zu vergessen ist auch die (Ganzkörper-)Fernbestrahlung mit der Weichstrahlmethode, die sich insbesondere für Erythrodermien



Abb. 5 R. G. Panizzon, J. S. Cooper: Radiation Treatment and Radiation Reactions in Dermatology. Heidelberg: Springer, 2004.

eignet. Hier gibt es nicht nur die Möglichkeit einer Bestrahlung am stehenden Patienten, sondern auch die von Jung entwickelte Methode beim liegenden Patienten.

Im Rahmen interdisziplinärer Konsultationen der dermatologischen Onkologie gibt es immer wieder die Möglichkeit, dass der Dermatologe die Radiotherapie erwähnt und hier, falls er nicht selbst die Radiotherapie durchführt, dies in Zusammenarbeit mit dem Radioonkologen bespricht. Es gibt auch immer wieder die Möglichkeit, dass jüngere Kollegen Röntgenapparate von älteren Kollegen übernehmen können. Diese Apparate funktionieren auch heute noch einwandfrei, müssen jedoch technisch überprüft und vom Radiophysiker regelmäßig anhand von Messungen kontrolliert werden. Dies sollte aber kein Hindernis sein mit solchen Geräten umgehen zu können. Beispiele solcher Geräte sind Namen wie Dermax, Dermophos, aber auch Therapix, Siemens Dermopan, Picker, die Geräte von Bucky, Siemens RT 50 bzw. 100 und General Electric. In den USA bestrahlen Dermatologen sehr oft nur noch mit Grenzstrahlgeräten, dies aus versicherungstechnischen Gründen. Mit diesen Geräten können immerhin viele oberflächliche Prozesse (bis 1 Millimeter Gewebehalbwerttiefe) bestrahlt werden. Ansonsten sind die heute zur Verfügung stehenden Geräte meist kombinierte Geräte, die Grenzstrahlen bis zur Tiefentherapie, d.h. von $10 \mathrm{kV}$ bis $150 \mathrm{kV}$ einschließen, z. B. das Gerät Darpac 150. 